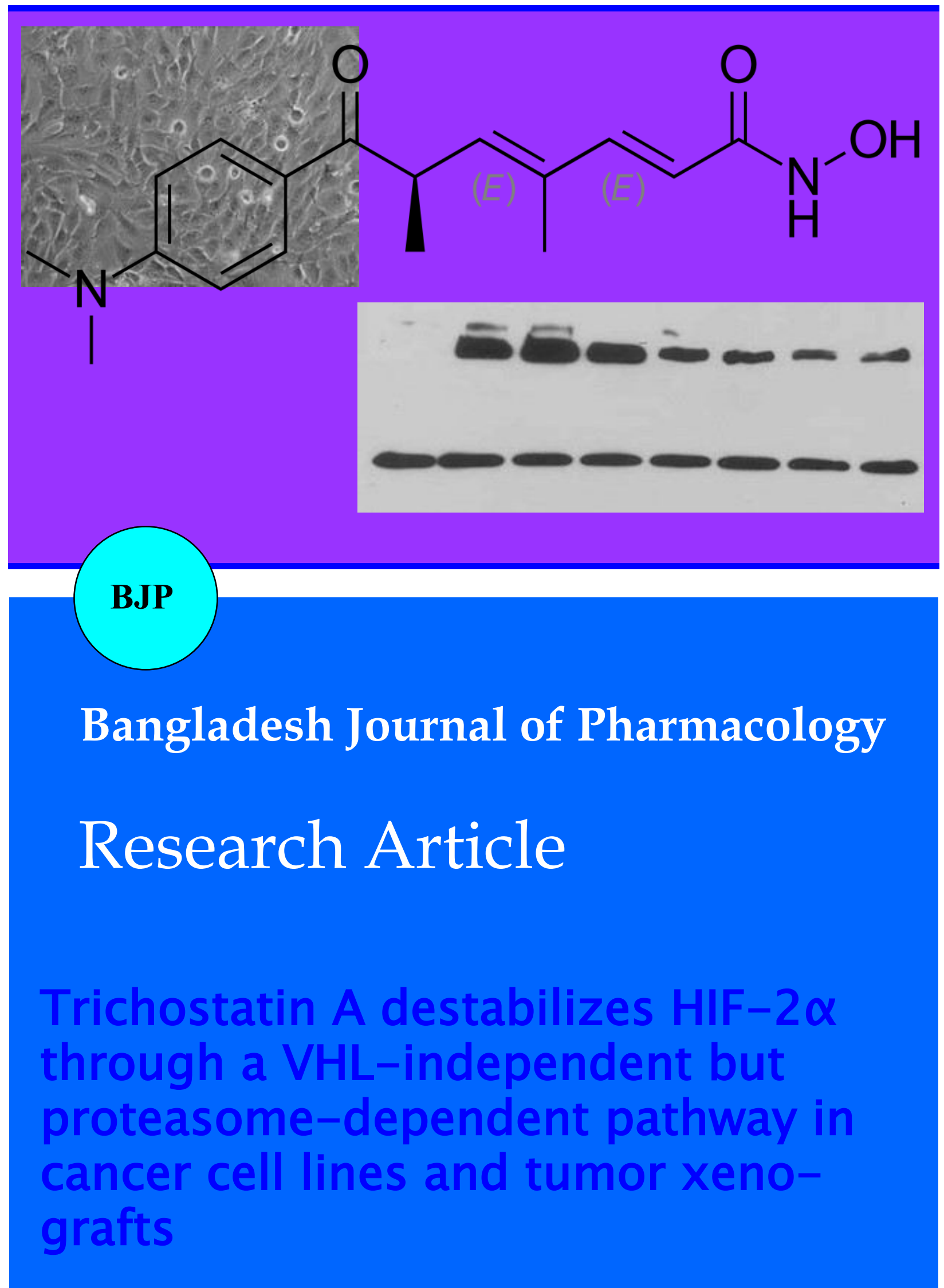




\title{
Trichostatin A destabilizes HIF-2 $\alpha$ through a VHL-independent but proteasome-dependent pathway in cancer cell lines and tumor xenografts
}

\author{
Qingcheng Yang, , Yuzhen Du², Dongdong Cheng1, Zhichang Zhang1 and Xinhui Du' \\ ${ }^{1}$ Department of Orthopedics, Sixth People's Hospital, Shanghai JiaoTong University, Shanghai 200233, China; \\ ${ }^{2}$ Department of Clinical Laboratory, Sixth People's Hospital, Shanghai Jiao Tong University, Shanghai 200233, \\ China.
}

\begin{tabular}{|c|c|}
\hline \multicolumn{2}{|l|}{ Article Info } \\
\hline Received: & 18 February 2 \\
\hline Accepted: & 25 February 201 \\
\hline Available Online: & 25 March 2 \\
\hline \multicolumn{2}{|c|}{ DOI: 10.3329/bjp.v8i2.13842 } \\
\hline \multicolumn{2}{|c|}{$\begin{array}{l}\text { Cite this article: } \\
\text { Yang Q, Du Y, Cheng D, Zhang Z, Du } \\
\text { X. Trichostatin A destabilizes HIF-2a } \\
\text { through a VHL-independent but } \\
\text { proteasome-dependent pathway in } \\
\text { cancer cell lines and tumor xeno- } \\
\text { grafts. Bangladesh J Pharmacol. 2013; } \\
\text { 8: } 142-48 \text {. }\end{array}$} \\
\hline
\end{tabular}

\begin{abstract}
Histone deacetylase (HDACs) inhibitors are a new generation of anti-cancer agents. Little is known regarding the effect of HDAC inhibitors on HIF-2a. The effect of trichostatin A (TSA), a class I/II HDAC inhibitor, on HIF-2a protein expression was investigated in cancer cell lines and tumor xenografts. Results showed TSA inhibited the HIF-2a protein expression in a dosedependent manner, which was VHL-independent, but proteasome-dependent in cell lines. In tumor xenografts, TSA inhibited tumor growth and HIF-2a expression. Knocking down of HDAC6 by small RNA interfering decreased HIF-2a protein expression. HDAC6 physically interracted with HIF-2a, and HIF-2 $\alpha$ was acetylated by TSA. TSA destabilizes HIF-2a in a proteasome dependent manner, which is unrelated to VHL, suggesting the anticancer effect of TSA is at least partially mediated by its inhibition of HIF-2a, which provides a new insight into the molecular mechanism underlying the anticancer effect of HDAC inhibitors.
\end{abstract}

\section{Introduction}

The survival of mammalian cells requires constant oxygen and nutrients. Precise homeostatic control has been developed to continuously adjust blood flow, which may maintain adequate oxygen tension, $\mathrm{pH}$, and appropriate supply of glucose and other nutrients (Carmeliet, 2000). However, in solid tumors, the tumor expansion exceeds the development of blood vessels, which results in a hypoxic microenvironment (Evans and Koch, 2003; Hockel and Vaupel, 2001). Hypoxia is not only a result of decrease in blood vessels, but also a consequence of the development of anatomically and functionally abnormal vasculatures (Vaupel, 2004). Blood vessels in tumors poorly develop, which are tortuous and leaky, and frequently contain dead ends due to the dysregulation of angiogenesis in tumors (Fraisi et al., 2009).
The activation of hypoxia-inducible factor-1 (HIF-1), a transcription factor, is one of the most important responses of tumors to hypoxia for survival. It has been widely accepted that HIF-1 functions as a master regulator in the molecular and responses to hypoxia (Semenza, 2009; Caro, 2001). HIF-1 is a heterodimer complex and consists of a constitutively expressed subunit (ARNT) and a subunit, of which the expression is regulated by oxygen levels. When oxygen is sufficient, HIF-a is hydroxylated at two prolyl residues in the oxygen-dependent degradation domain (ODD) by prolyl hydroxylases (PHD), which allows the binding of VHL, an E3 ubiquitin ligase. Under this condition, HIF-a is ubiquitinated and targeted for subsequent proteasome degradation (Semenza, 2000). In addition to hypoxia, HIF-a expression has been shown to be regulated by various growth factors, hormones, oncogenes and tumor suppressors (Semenza, 
2000). There are two structurally related HIF factors termed HIF-1a and HIF-2a. Despite their sequence homology and identical DNA-binding sites, both HIF$1 \alpha$ and HIF-2 $\alpha$ have distinct distributions and appear to activate different genes, thus playing critical but distinct roles in tumor progression (Qing and Simon, 2009). Previous studies have demonstrated that HIF-1 and HIF-2 are over-expressed in some primary and metastatic tumors and their expression is closely correlated with adverse prognosis and poor response to chemotherapy and/or radiotherapy (Semenza, 2008; Bertout et al., 2008; Semenza, 2010).

HIF-1a and HIF-2a exert effects on cancer progression by binding to and activating target genes with cis-acting hypoxia response elements containing the consensus binding site 5'-RCGTG-3'. A variety of genes that are induced by hypoxia in an HIF-1-dependent manner encode proteins which have key roles in the cancer biology, including angiogenesis, glucose metabolism, microenvironment remodeling and other changes required for tumor progression (Semenza, 2010). Because of its pivotal roles in tumor progression, HIF has been considered as a potential target for cancer therapy, and many HIF inhibitors have been screened and tested in cancers (Melillo, 2007).

Histone deacetylase (HDAC) inhibitors are a relatively new class of anti-cancer agents that play important roles in the regulation of epigenesis and non-epigenesis including death, apoptosis and cell cycle arrest in cancer cells. Recently, HDAC inhibitors have been clinically applied in cancer patients and two HDAC inhibitors have been approved by the FDA. However, the molecular mechanisms underlying the therapeutic effect of HDAC inhibitors on cancers are not fully understood (Kim and Bae, 2011). Recent findings indicate that several HDAC inhibitors can inhibit the angiogenesis, a process essential for tumor metabolism and progression. Accumulating evidence supports that the inhibition of angiogenesis is at least partially mediated by HIF (Chen and Sang, 2011; Liang et al., 2006). Previous studies have shown that HDAC inhibitors not only destablize HIF-1a protein expression (Qian et al., 2004; Qian et al., 2006; Kong et al., 2006), but inhibit the HIF-1a transcriptional activity (Fath et al., 2006). However, little is known regarding the effect of HDAC inhibitors on HIF-2a. In the present study, our results showed that HDAC inhibitor TSA could induce HIF-2a degradation in a VHL-independent but proteasome-dependent pathway, which is similar as the degradation of HIF-1a mediated by HDAC inhibitors.

\section{Materials and Methods}

Cell culture

Cell lines MG-63, 786-0, HT1080 and HeLa were purchased from American Type Culture Collection (ATCC). Cells were cultured in Dulbecco's modified Eagle's medium (DMEM) containing L-glutamine, sodium pyruvate, $10 \%$ fetal bovine serum (FBS), penicillin $(100 \mathrm{U} / \mathrm{mL})$ and streptomycin $(100 \mu \mathrm{g} / \mathrm{mL})$. Cells were grown in a humidified environment containing $5 \% \mathrm{CO}_{2}$ at $37^{\circ} \mathrm{C}$. Hypoxia condition was introduced by using a hypoxic chamber (In $\mathrm{VIVO}_{2}$; Ruskin Tech).

\section{Reagents}

Common chemicals, solvents, general reagents and Cyclophosphamide (CTX) were purchased from Sigma. TSA and proteasome inhibitor MG132 were from Biomol (Plymouth Meeting, PA).

\section{Western blot assay and immunoprecipitation}

Polyclonal antibody against HIF-2a was from Novus Biologicals (USA). Monoclonal antibodies against Hdac4, Hdac6, Hdac7 and a-tubulin were from Santa Cruz Biotechnology (USA). Monoclonal anti-acetyllysine antibody was from Cell Signaling (Canada). Monoclonal antibody against FLAG tag was from Sigma. Horseradish peroxidase - conjugated secondary antibodies were from Sigma and in vitrogen.

Cell extracts for Western blot assay were obtained by homogenizing cells in $8 \mathrm{M}$ urea with a portable homogenator, which has been described previously (Kong et al., 2006). For Western blot assay, $40 \mu \mathrm{g}$ of cell extracts were loaded onto 7.5\%SDS-polyacrylamide gels, and proteins were then transferred onto PVDF membranes (Bio-Rad, CA) which were blocked in 5\% non-fat milk in TBST followed by incubation overnight at $4^{\circ} \mathrm{C}$ with indicated antibodies. Immunolabeling was done with an ECL plus kit from Amersham. For immunoprecipitation, cells were lysed on ice for 1 hour in Ripa lysis buffer with $1 \times$ protease inhibitor, followed by two freeze-thaw-vortex cycles. After centrifugation at 15,000x $\mathrm{g}$ for $10 \mathrm{~min}$, supernatants were diluted with lysis buffer and incubated with primary antibody for 4 hours at $4^{\circ} \mathrm{C}$ with agitation, followed by precipitation with protein A-Sepharose (Pierce). Immunoprecipitates were washed thoroughly with lysis buffer and subjected to immunoblotting.

\section{siRNAs, plasmids and transfections}

SMARTpool siRNAs against Hdac4, Hdac6, Hdac7 and control siRNA were from Dharmacon. siRNA transfection was performed by using the DharmaFECT according to the manufacture's instructions. pCMV3FLAG-1 vector was from Stratagene (South San Francisco, CA), HIF-2a full-length insert sequence was generated by PCR using HT1080 cDNA as the template and introduced to the pCMV-3FLAG-1 vector at the BamH1 and Sal1 restriction sites. FLAG-HIF-2a P405A/ P531A mutant plasmid was generated by using a site directed mutagenesis kit from Stratagene (South San Francisco, CA), and all the plasmids were validated by 
DNA sequencing. Plasmid transfection was carried out in the presence of Lipofectamine 2000 (Invitrogen) according to the manufacture's instructions.

\section{RNA isolation and qPCR to analyze mRNA levels}

Total RNA was isolated from cells with Trizol Reagent (Invitrogen). RNA was reversely transcribed using random hexamers and SuperScript III reverse transcriptase (in vitrogen). Levels of mRNA expression were quantified by SYBR Green quantitative reverse transcription -PCR (qPCR) using a 7900 HT real-time system (Applied Biosystems). All primer sets designed for detection of target gene mRNA by SYBR Green qPCR were validated for their product specificity and amplification efficiency using melt curve analysis, qPCR product sequencing and standard dilution analysis. Primer sequences are as the following: HIF-2 a sense: 5' CCGTACTGTCAACCTCAAGTC 3', antisense: 5' CCTTGTAGCCACACAGACTATT 3'. EPO sense: 5'CAAGGAGGCCGAGAATATCAC3', antisense: 5' CATCCTCTTCCAGGCATAGAAAT 3'. PAI1 sense: 5' AGATCGAGGTGAACGAGAGT3', antisense: 5' CACAAAGAGGAAGGGTCTGTC3', and beta-actin sense: 5' ACCCAGCACAATGAAGATCA 3', antisense: 5'CGATCCACACGGAGTACTTG3'. qPCR results were normalized with beta-actin. Results are the averages of a minimum of three independent experiments performed in triplicate.

\section{Tumor xenograft in vivo}

Athymic 6-8 week-old BALB/c nude mice were purchased from the Animal Center of Shanghai Jiaotong University, China. Osteosarcoma cell line MG-63 cells were trypsinized and washed with PBS. Then, $3 \times 10^{6}$ cells were re-suspended in $200 \mu$ Lof PBS and injected subcutaneously at the right flank of nude mice. One week later, subcutaneous tumors were macroscopic visible. Cancer-bearing mice were randomly divided into control group $(n=6)$, TSA group $(n=6)$ and cyclophosphamide (CTX) group $(n=6)$. CTX served as a positive control of anticancer agent. Mice in the TSA group were treated with TSA at $1 \mathrm{mg} / \mathrm{kg}$ once biweekly; mice in the CTX group were treated with CTX at $20 \mathrm{mg} / \mathrm{kg}$ once biweekly; and mice in the control group were treated with normal saline containing 5\%DMSO. Mice were treated for 17 days, tumor size was measured with a caliper every three days, and the tumor volume calculated as $\mathrm{V}=\mathrm{W}^{\wedge} 2 \mathrm{XL} / 2$. The therapeutic efficacy of TSA and CTX was assessed by the change in tumor volume. After the treatment, mice were sacrificed and tumors were harvested for Western blot assay.

\section{Statistical analysis}

Comparisons of unpaired data were done with Student's t-test. A value of $\mathrm{p}<0.05$ was considered statistically significant.

\section{Results}

To investigate the effect of TSA on HIF-2a protein expression, MG-63 cells were treated with TSA at

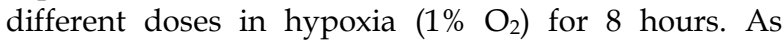
shown in Figure 1A, TSA induced a dose-dependent inhibition of HIF-2a protein expression. Similar phenomena were observed in other cell lines (data not shown). TSA did not affect the HIF-2a mRNA expression but remarkably inhibited the expression of HIF-2a specific target genes such as EPO and PAI1 (Figure 1B). To rule out the effect of TSA on HIF-2a translation, MG63 cells were treated with MG132 without or with TSA (300 nM). Results showed that HIF-2a protein accumulation was not affected by TSA. Collectively, these findings suggest that TSA inhibits HIF-2a protein expression via a posttranslational mechanism.

To examine whether TSA-mediated HIF-2a degradation is related to proteasome, MG-63 cells were treated in hypoxia for 8 hours with TSA at different concentrations in the absence or presence of $5 \mu \mathrm{M}$ proteasome inhibitor MG132. As shown in Figure 2A, MG132 completely reversed the effect of TSA on HIF-2a, indicating that TSA mediated HIF-2 $\alpha$ degradation was proteasome-dependent. To test the role of VHL in the TSA induced HIF-2a degradation, VHL deficient cells (786-0 cells) were treated with TSA without or with MG132. As shown in Figure 2B, TSA inhibited the HIF$2 \alpha$ expression in VHL (-/-) cells in a dose-dependent manner as in VHL $(+/+)$ cells, and this effect was also abolished by MG132. These findings suggest that the degradation is VHL-independent. To confirm this observation further, MG-63 cells were transfected with Flag-tagged plasmids encoding either full-length HIF$2 a$ or HIF-2a mutant (P405A/P531A). As shown in Figure $2 \mathrm{C}$, mutations of two key proline residues in ODD affect the response to TSA. Taken together, these did not findings indicate that TSA-induced HIF-2a degradation is proteasome-dependent, but independent of VHL.

To evaluate the effect of TSA on tumor growth in vivo, MG-63 cells were injected subcutaneously into nude mice to prepare a tumor xenograft. One week later, mice were randomly divided into control, TSA and CTX group, in which mice were treated with normal saline, TSA and CTX, respectively. Tumor growth curve (Figure 3A) showed that TSA and CTX significantly inhibited the tumor growth $(p=0.02)$. No significant difference in body weight was noticed between TSA group and control group, and the toxic effects were not observed in both groups. However, in the CTX group, the body weight was significantly lower than that in the control group (data not shown).

To assess if the in vivo inhibitory effect of TSA is related to its effect on HIF-2a, the HIF-2a protein expression was measured in tumor xenografts. Western blot assay 


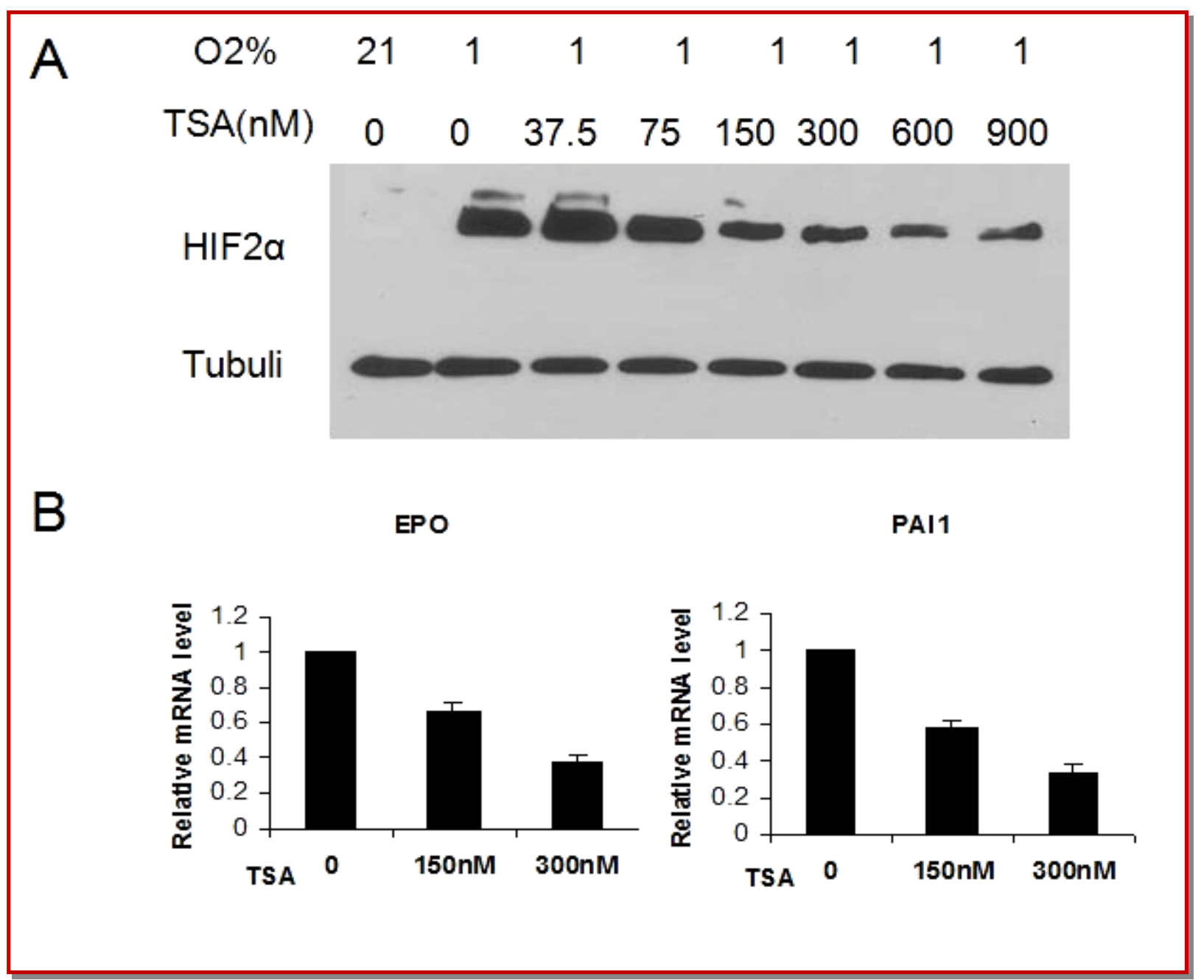

Figure 1: Effect of TSA on protein and mRNA expression of HIF-2 $a$

(A) MG-63 cells were exposed to 1\% oxygen in the presence of TSA at different concentrations for 8 hours, and cell extracts were subjected to SDSPAGE followed by immunoblotting. (B) VHL deficient (-/-) 786-0 cells were treated with TSA at different concentrations for 16 hours in normoxia, total RNA was isolated and qRT-PCR was performed to detect the relative mRNA expression levels of HIF-2a, HIF-2a specific target genes EPO and PAI1

showed that TSA remarkably inhibited the HIF-2a expression in vivo (Figure $3 \mathrm{~B}$ ), implying that the antitumor effect of TSA is at least partially mediated by its suppression on HIF-2a expression.

Class II histone deacetylases HDAC4, HDAC6 and HDAC7 have been reported to be implicated in the regulation of HIF-1a stability (Qian et al., 2006; Kong et al., 2006). Thus, we hypothesized that class II HDACs may involve in the regulation of HIF-2a. To test this hypothesis, the expression of HDAC4, HDAC6 and HDAC7 was down-regulated by RNA interfering. Results showed that silencing of HDAC6 decreased the HIF-2a protein expression in MG-63 cells (Figure 4A). However, the HIF-2a protein expression was not affected by silencing of HDAC4 or HDAC7 (data not shown). These findings suggest that HDAC6 is likely to be involved in the HIF-2a protein stability. To explore the physical interaction between HIF-2a and HDAC6, immunoprecipitation of HDAC6 was done with HDAC6 antibody. The precipitated complexes were immunoblotted for HIF-2a. As shown in Figure 4B, HIF $-2 \alpha$ was detectable in the precipitated complexes, confirming that HIF-2a can physically interact with HDAC6.

In addition, we investigated if TSA directly acetylates HIF-2a. MG-63 cells were treated with proteasome inhibitor MG132 in hypoxia for $6 \mathrm{~h}$ with or without 600 nM TSA. Results showed MG132 prevented the TSAmediated HIF-2a degradation. Cell lysates were immunoprecipitated with HIF-2a antibody. As shown in Figure $4 C$, the acetylated lysine was detected in the immunoprecipitated HIF-2a after TSA treatment, indicating that TSA directly acetylates HIF-2a. 


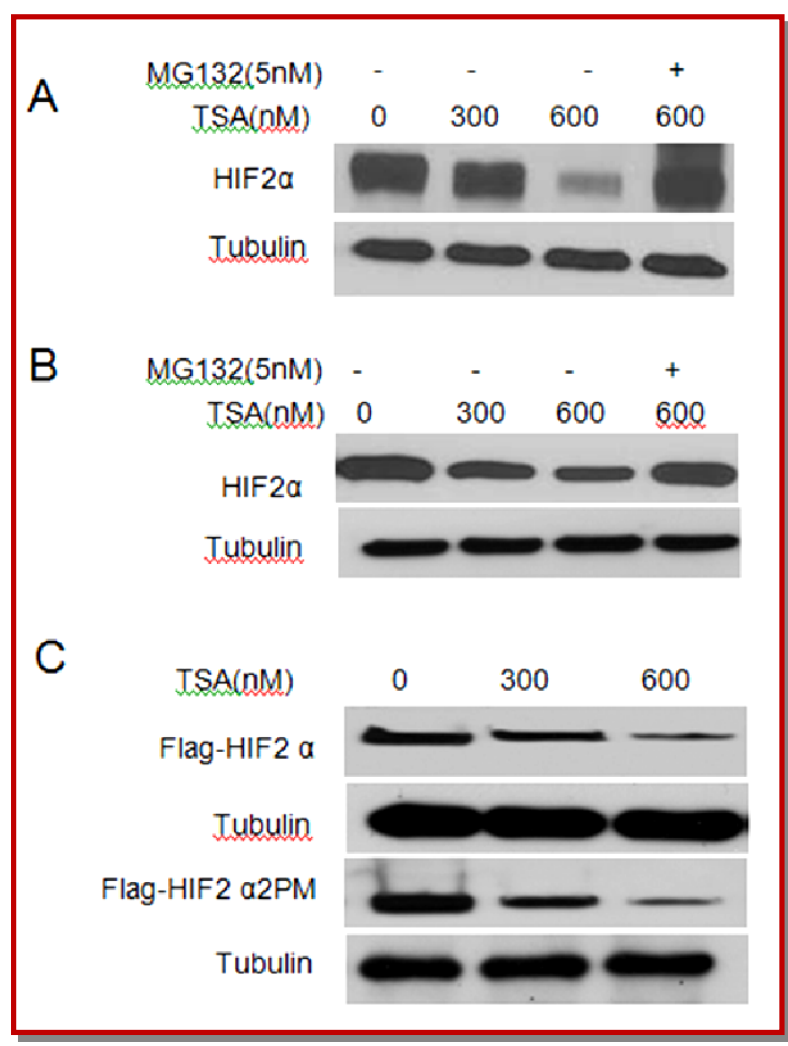

Figure 2: TSA-induced HIF-2a degradation is proteasome dependent but VHL-independent

(A) MG-63 cells were treated in hypoxia $\left(1 \% \mathrm{O}_{2}\right)$ for 8 hours in the presence of TSA at 300 or $600 \mathrm{nM}$ without or with $5 \mu \mathrm{M}$ proteasome inhibitor MG132. Cell extracts were subjected to western blot assay for HIF-2a and tubulin. (B) VHL deficient (-/-) 786-0 cells were treated with TSA at different concentrations without or with $5 \mu \mathrm{M}$ proteasome inhibitor MG132 in normoxia for 8 hours. Cell extracts were subjected to western blot assay for HIF-2a and tubulin. (C) $5 \mu \mathrm{g}$ of flag-tagged wild type HIF -2a plasmid or prolyl residues 405 and 531 mutant (HIF-2a 2PM) were transfected into MG-63 cells at $80 \%$ cell confluence with Lipofectamine 2000. One day later, cells were evenly split into 3 plates and exposed to hypoxia $\left(1 \% \mathrm{O}_{2}\right)$ for 8 hours with TSA at different concentrations, and cells extracts were subjected to immunoblotting with anti-flag and antitubulin antibodies

\section{Discussion}

HDACs are a large family of enzymes which can remove the acetyl group from the $\mathrm{N}-\varepsilon$-lysines of histones. To date, 18 human HDAC have been identified and can be classified into 4 groups based on their homology with yeast HDACs (de Ruijter et al., 2003; Blander and Guarente, 2004). Histone acetylation or deacetylation is thought to facilitate or inhibit gene transcription. Besides regulating histone modification, HDACs can also regulate the post-translational acetylation of many non-histone proteins, including transcription factors, chaperones and signaling molecules (Glozak et al., 2005). The acetylation of these proteins is usually reversibly regulated by the dynamic balance between acetyl transferases (HATs) and HDACs. A large number of HDAC inhibitors have been purified

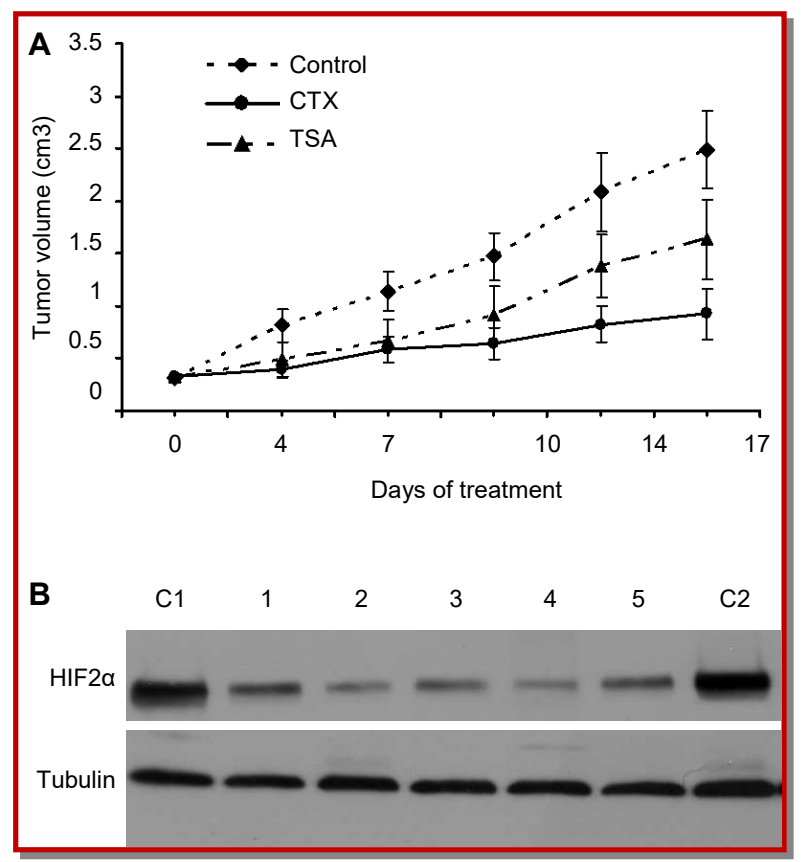

Figure 3: Effect of TSA on tumor growth and HIF-2a expression in vivo

(A) MG-63 cells were inoculated into nude mice to induce subcutaneous tumors. One week later, mice were randomly divided into control, TSA and CTX groups, in which mice were treated with normal saline (control group), TSA and CTX, respectively, for 17 days. Data were expressed as mean \pm standard error (SEM). (B) Tumors were harvested and homogenized; protein was subjected to SDS-PAGE followed by immunoblotting for HIF-2a and tubulin. C1, C2: control group; 1, 2, 3, 4, 5: TSA-treated group

from natural sources, or synthesized. The mechanisms of action of HDAC inhibitors are thought to be related to the alteration of gene expression and the changes in non-histone proteins at epigenetic and post-translational levels, respectively (Kim and Bae, 2011).

TSA is the first natural hydroxamate found to inhibit class I and II HDACs exhibiting powerful anti-cancer effect via inhibiting tumor angiogenesis, which may be related to the suppression of HIF-1a (Kim et al., 2001). The effect of HDAC inhibitors on HIF-1a has been studied extensively. In the current study, the effect of TSA on HIF-2a was investigated. Our findings indicated that TSA destabilized HIF-2 $\alpha$ in a VHL-independent, but proteasome-dependent way. In cancer-bearing nude mice, TSA significantly inhibited the tumor growth, and more importantly, it destabilized HIF-2a in vivo, suggesting that the anti-cancer effect of TSA is at least partially mediated by its inhibitory effect on HIF$2 a$. In fact, the role of HIF-2a in tumor progression has attracted more attentions in recent years for the following reasons. First, although HIF-1a and HIF-2a are highly homologous, increasing evidence suggests they have distinct transcriptional targets and different influences on tumor growth. Furthermore, the nontranscriptional effects of HIF-a subunits, including 


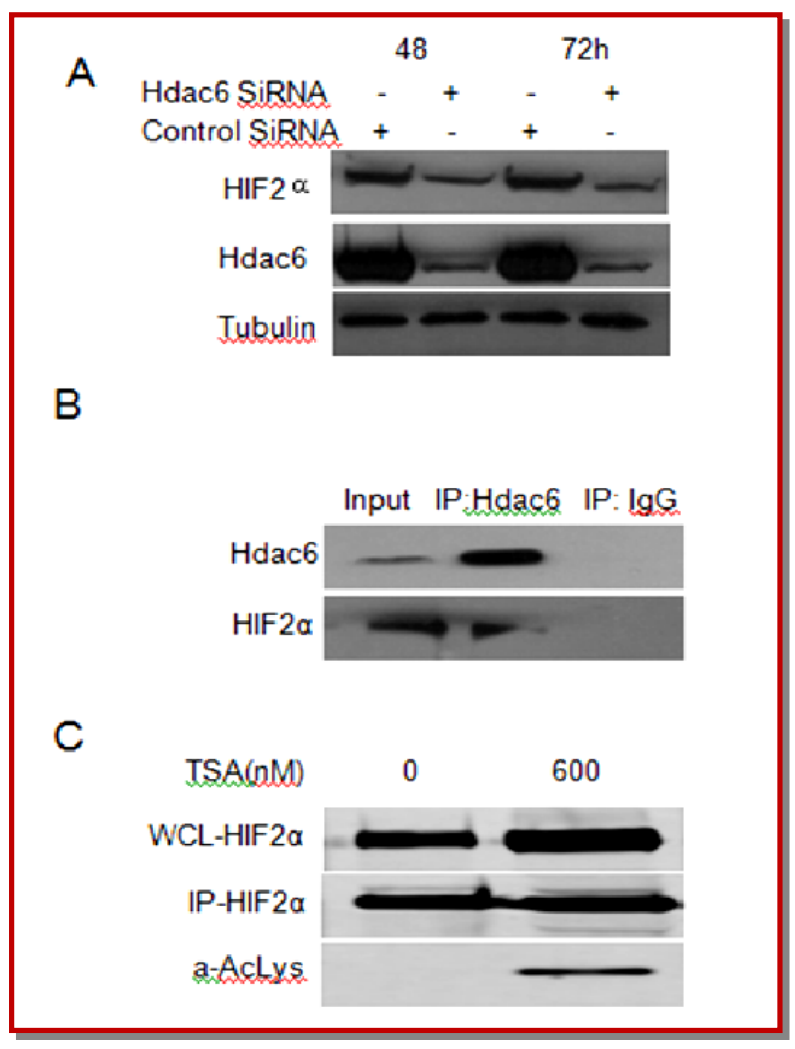

Figure 4: HDAC6 is involved in regulation of HIF-2a degradation and HIF-2 $\alpha$ is acetylated by TSA

(A) siRNA against HDAC6 and control siRNA were transfected into MG-63 cells at $50 \mathrm{nM}$. At 48 and 72 hours after transfection, cells were exposed to hypoxia for 8 hours, and cell extracts were subjected to western blot assay for HIF-2a, HDAC6 and tubulin. (B) MG-63 cells were treated for 8 hours in hypoxia $\left(1 \% \mathrm{O}_{2}\right)$, and cell extracts were immunoprecipitated with antibody against either HDAC6 or control IgG. The immunocomplexes were detected for HDAC6 and HIF-2a by western blot assay, and $20 \mu \mathrm{g}$ of cell extracts without immunoprecipitation served as a control. (C) MG-63 cells were exposed to hypoxia $\left(1 \% \mathrm{O}_{2}\right)$ and MG132 $(5 \mu \mathrm{M})$ for 6 hours with or without $600 \mathrm{nM}$ TSA, and cell lysates were immunoprecipitated with anti-HIF2a antibody. Cell lysates and immunocomplexes were immunoblotted with anti-HIF-2a and antiacetyl-lysine (a-AcLys) antibodies

effects on Notch and c-Myc pathways, contribute to their distinct functions (Qing and Simon, 2009; Patel and Simon, 2008). The transcriptional targets of HIF-2a include genes involved in the erythropoiesis, angiogenesis, metastasis, and proliferation. Therefore, HIF-2a plays crucial roles in both physiology and tumorigenesis (Qing and Simon, 2009; Patel and Simon, 2008). Second, in some cancers such as neuroblastoma and renal clear cell carcinoma, HIF-2a appears to play more important roles than HIF-1a (HolmquistMengelbier et al., 2006; Mack et al., 2003). Our findings suggest that TSA can inhibit both HIF-1a and HIF-2a protein expression, which provides a new insight into the molecular mecha-nisms underlying the anti-cancer effect of HDAC inhibitors.

The mechanism underlying the degradation of HDAC inhibitor-mediated HIF-a still remains unclear. In the case of HIF-1a, a couple of mechanisms have been proposed including direct HIF-1a acetylation at K532 site, the disruption of chaperone protein (HSP70 and HSP90) activities and the involvement of tubulin acetylation (Chen and Sang, 2011). However, its exact mechanism is still controversial (Chen and Sang, 2011). By immunoprecipitation, our results showed that HIF$2 a$ was acetylated by TSA. However, a recent observation from Dioum et al indicated that Sirt1, a class III HDAC, was implicated in the regulation of HIF $-2 \alpha$ signaling, and HIF-2a acetylation was not associated with HIF-2a stability (Dioum et al., 2009). In previous studies, HDAC4 and HDAC6 have been demonstrated to be involved in the regulation of HIF1a stability (Qian et al., 2006; Kong et al., 2006). In the present study, our findings revealed that HDAC6 was likely to be implicated in the regulation of HIF-2a stability. HDAC6 has been linked to the functions of chaperon HSP90 (Chen and Sang, 2011), and inhibition of HDAC6 may interfere with the chaperon HSP90 activity, leading to the degradation of its client proteins. Therefore, more work is needed to determine the role of HSP90 in TSA induced HIF-2a degradation.

In summary, our findings suggest that TSA can destabilize HIF-2a both in vitro and in vivo, which is proteasome-dependent, but VHL independent. These results provide a new insight into the molecular mechanism underlying the anti-cancer effect of HDAC inhibitors.

\section{Contribution}

Drs. Qingcheng Yang and Yuzhen Du contributed equally in this work.

\section{Acknowledgement}

This work was partially supported by grant from Chinese Natural Science Foundation (No. 30973017 to Y.Q.).

\section{References}

Bertout JA, Patel SA, Simon MC. The impact of oxygen availability on human cancer. Nat Rev Cancer. 2008; 8: 96775 .

Blander G, Guarente L. The Sir2 family of protein deacetylases. Annu Rev Biochem. 2004; 73: 417-35.

Carmeliet P. Mechanisms of angiogenesis and arteriogenesis. Nature Med. 2000; 6: 389-95.

Caro J. Hypoxia regulation of gene transcription. High Alt Med Biol. 2001; 2: 145-54.

Chen S, Sang N. Histone deacetylase inhibitors: The epigenetic therapeutics that repress hypoxia-inducible factors. J Biomed Biotechnol. 2011; 2011: 197946.

de Ruijter AJ, van Gennip $\mathrm{AH}$, Caron $\mathrm{HN}$, Kemp S, van 
Kuilenburg AB. Histone deacetylases (HDACs): Characterization of the classical HDAC family. Biochem J. 2003; 370: 737-49.

Dioum EM, Chen R, Alexander MS, Zhang Q, Hogg RT, Gerard RD, Garcia JA. Regulation of hypoxia-inducible factor 2alpha signaling by the stress-responsive deacetylase sirtuin 1. Science. 2009; 324: 1289-93.

Evans SM, Koch CJ. Prognostic significance of tumor oxygenation in humans. Cancer Lett. 2003; 195: 1-16.

Fath DM, Kong X, Liang D, Lin Z, Chou A, Jiang Y, Fang J, Caro J, Sang N. Histone deacetylase inhibitors repress the transactivation potential of hypoxia-inducible factors independently of direct acetylation of HIF-alpha. J Biol Chem. 2006; 281: 13612-19.

Fraisi P, Mazzone M, Schmidt T, Carmeliet P. Regulation of angiogenesis by oxygen and metabolism. Dev Cell. 2009; 16: 97-106.

Glozak MA, Sengupta N, Zhang X, Seto E. Acetylation and deacetylation of non-histone proteins. Gene 2005; 363: 15-23.

Hockel M, Vaupel P. Biological consequences of tumor hypoxia. Semin Oncol. 2001; 28: 36-41.

Holmquist-Mengelbier L, Fredlund E, Lofstedt T, Noguera R, Navarro S, Nilsson H. Recruitment of HIF-1alpha and HIF2alpha to common target genes is differentially regulated in neuroblastoma: HIF-2alpha promotes an aggressive phenotype. Cancer Cell. 2006; 10: 413-23.

Kim HJ, Bae SC. Histone deacetylase inhibitors: Molecular mechanisms of action and clinical trials as anti-cancer drugs. Am J Transl Res. 2011; 3: 166-79.

Kim MS, Kwon HJ, Lee YM, Baek JH, Jang JE, Lee SW, Moon EJ, Kim HS, Lee SK, Chung HY, Kim CW, Kim KW. Histone deacetylases induce angiogenesis by negative regulation of tumor suppressor genes. Nat Med. 2001; 7: 437-43.

Kong X, Lin Z, Liang D, Fath D, Sang N, Caro J. Histone deacetylase inhibitors induce VHL and ubiquitinindependent proteasomal degradation of hypoxia-inducible factor 1alpha. Mol Cell Biol. 2006; 26: 2019-28.
Liang D, Kong X, Sang N. Effects of histone deacetylase inhibitors on HIF-1. Cell Cycle. 2006; 5: 2430-35.

Mack FA, Rathmell WK, Arsham AM, Gnarra J, Keith B, Simon MC. Loss of pVHL is sufficient to cause HIF dysregulation in primary cells but does not promote tumor growth. Cancer Cell. 2003; 3: 75-88.

Melillo G. Hypoxia-inducible factor 1 inhibitors. Methods Enzymol. 2007; 435: 385-402.

Patel SA, Simon MC. Biology of hypoxia-inducible factor2alpha in development and disease. Cell Death Differ. 2008; 15: 628-34.

Qian DZ, Kachhap SK, Collis SJ, Verheul HM, Carducci MA, Atadja P, Pili R. Class II histone deacetylases are associated with VHL-independent regulation of hypoxia-inducible factor 1 alpha. Cancer Res. 2006; 66: 8814-21.

Qian DZ, Wang X, Kachhap SK, Kato Y, Wei Y, Zhang L, Atadja P, Pili R. The histone deacetylase inhibitor NVPLAQ824 inhibits angiogenesis and has a greater antitumor effect in combination with the vascular endothelial growth factor receptor tyrosine kinase inhibitor PTK787/ZK222584. Cancer Res. 2004; 64: 6626-34.

Qing G, Simon MC. Hypoxia inducible factor-2alpha: A critical mediator of aggressive tumor phenotypes. Curr Opin Genet Dev. 2009; 19: 60-65.

Semenza GL. Defining the role of hypoxia-inducible factor 1 in cancer biology and therapeutics. Oncogene. 2010; 29: 62534 .

Semenza GL. Expression of hypoxia-inducible factor 1: Mechanisms and consequences. Biochem Pharm. 2000; 59: 47-53.

Semenza GL. Hypoxia-inducible factor 1 and cancer pathogenesis. IUBMB Life. 2008; 60: 591-97.

Semenza GL. Regulation of oxygen homeostasis by hypoxiainducible factor 1. Physiology (Bethesda). 2009; 24: 97-106

Vaupel P. Tumor microenvironmental physiology and its implications for radiation oncology. Semin Radiat Oncol. 2004; 14: 198-206.
Author Info
Qingcheng Yang (Principal contact)
e-mail: tjyqc@163.com 